\title{
THE PAN-PACIFIC PLANET SEARCH. II. CONFIRMATION OF A TWO-PLANET SYSTEM AROUND HD 121056
}

\author{
Robert A. Wittenmyer ${ }^{1,2}$, Liang Wang ${ }^{3}$, Fan Liu $^{4}$, Jonathan Horner $^{2,5}$, Michael EndL $^{6}$, \\ JOHN AsHer JOHNSON ${ }^{7}$, C.G. TINNEY ${ }^{1,2}$, AND B.D. CARTER ${ }^{5}$ \\ ${ }^{1}$ School of Physics, University of New South Wales, Sydney, NSW 2052, Australia; rob@unsw.edu.au \\ 2 Australian Centre for Astrobiology, University of New South Wales, Sydney, NSW 2052, Australia \\ ${ }^{3}$ Key Laboratory of Optical Astronomy, National Astronomical Observatories, Chinese Academy of Sciences, \\ A20 Datun Road, Chaoyang District, Beijing 100012, China \\ ${ }^{4}$ Research School of Astronomy and Astrophysics, Australian National University, Cotter Road, Weston Creek, ACT 2611, Australia \\ ${ }^{5}$ Computational Engineering and Science Research Centre, University of Southern Queensland, Toowoomba, Queensland 4350, Australia \\ ${ }^{6}$ McDonald Observatory, University of Texas at Austin, 1 University Station C1400, Austin, TX 78712, USA \\ ${ }^{7}$ Harvard-Smithsonian Center for Astrophysics, Cambridge, MA 02138, USA \\ Received 2014 November 18; accepted 2014 December 19; published 2015 February 10
}

\begin{abstract}
Precise radial velocities from the Anglo-Australian Telescope (AAT) confirm the presence of a rare short-period planet around the K0 giant HD 121056. An independent two-planet solution using the AAT data shows that the inner planet has $P=89.1 \pm 0.1$ days, and $\mathrm{m} \sin i=1.35 \pm 0.17 \mathrm{M}_{\mathrm{Jup}}$. These data also confirm the planetary nature of the outer companion, with $\mathrm{m} \sin i=3.9 \pm 0.6 \mathrm{M}_{\mathrm{Jup}}$ and $a=2.96 \pm 0.16 \mathrm{AU}$. HD 121056 is the most-evolved star to host a confirmed multiple-planet system, and is a valuable example of a giant star hosting both a short-period and a long-period planet.
\end{abstract}

Key words: planetary systems - stars: individual (HD 121056) - techniques: radial velocities

\section{INTRODUCTION}

With the discovery of more than 700 extrasolar planets by the radial-velocity method, and several thousand planet candidates by the Kepler spacecraft (Borucki et al. 2010; Batalha et al. 2013), the past $20 \mathrm{yr}$ have seen tremendous growth in our understanding of the startling diversity of planetary systems in the solar neighborhood. At the same time, planet-search efforts are now expanding into new realms of parameter space, seeking to understand how the detailed properties of planetary systems depend on the properties of their host stars. At the low-mass end, $\mathrm{M}$ dwarfs are being targeted by a number of optical and near-infrared radial-velocity surveys searching for rocky and potentially habitable planets (e.g., Endl et al. 2006; Quirrenbach et al. 2010; Bean et al. 2010; Johnson et al. 2010b; Mahadevan et al. 2012; Barnes et al. 2012; Bonfils et al. 2013; Wright et al. 2014). Meanwhile, virtually everything we know about planetary systems orbiting stars more massive than our Sun has come from taking advantage of stellar evolution. Highmass stars on the main sequence are too hot and rotate too rapidly (Galland et al. 2005) for the radial-velocity technique to work. By observing higher-mass stars when they evolve off the main sequence into subgiants and giants, precision velocity measurements become possible. The stars expand and cool, presenting an abundance of narrow spectral absorption lines for accurate velocity determination. This approach has been successfully used by several teams in recent years (e.g., Setiawan et al. 2003; Hatzes et al. 2005; Sato et al. 2005; Johnson et al. 2006; Döllinger et al. 2007; Niedzielski et al. 2009a; Wittenmyer et al. 2011a).

One result arising from these studies of evolved stars is a relative deficit of short-period planets, despite obvious selection biases in favor of detecting them. Several thousand subgiant and giant stars are being monitored by both Kepler and the programs mentioned above, but only seven planets are known to orbit giant stars $(\log g<3.5)$ within $a<0.5$ AU. They are HD 102956b ( $a=0.0810$ AU; Johnson et al. 2010a), Kepler-91b ( $a=0.072$ AU; Lillo-Box et al. 2014), KOI-1299b
( $a=0.3034 \mathrm{AU}$; Ciceri et al. 2014) and the two-planet systems orbiting Kepler-391 $\left(a_{b}=0.0744 \mathrm{AU}, a_{c}=0.1465 \mathrm{AU}\right.$; Rowe et al. 2014) and Kepler-56 ( $a_{b}=0.1028 \mathrm{AU}, a_{c}=0.1652 \mathrm{AU}$; Huber et al. 2013). The apparent shortfall of planets orbiting evolved stars has been noted by Johnson et al. (2007) and Sato et al. (2010). Two possible explanations are that either the planets are absent, or are swallowed by the host star as it expands (Kunitomo et al. 2011; Villaver et al. 2014).

From 2009 to 2014, the Pan-Pacific Planet Search (PPPS) observed 170 southern hemisphere subgiants and first-ascent giants (Wittenmyer et al. 2011a). The PPPS targets are redder than those observed by most surveys (Mortier et al. 2013) -we have chosen stars with $1.0 \leqslant(B-V) \leqslant 1.2$, whereas other surveys enforce $(B-V) \leqslant 1.0$. This color selection makes the PPPS targets complementary to the $\sim 450$ Northern "retired A stars" from the well-established Lick and Keck program (Johnson et al. 2006, 2011a). A complete target list is given in Wittenmyer et al. (2011a). HD 121056 (HIP 67851, HR 5224) is a bright southern $(V=6.19$, R.A. 13:53:52.06, decl. $-35: 18: 51.7)$ giant targeted by both the PPPS and the EXPRESS project (Jones et al. 2011), a similar radial-velocity campaign aimed at detecting planets orbiting southern hemisphere evolved stars. Recently, Jones et al. (2014) announced the discovery of two companions orbiting HD 121056: a rare short-period planet at $P=88.8$ day and an unconstrained massive outer body with an orbital period exceeding their observational baseline of 1523 days (4.2 yr).

In this paper, we present independent data that confirm both the inner planet and the planetary nature of the outer body in the HD 121056 system. Section 2 briefly describes the observational data and gives the stellar parameters. In Section 3, we detail the orbit-fitting process and give the planetary parameters. Finally, in Section 4, we give our conclusions and place this discovery in context.

\section{AAT OBSERVATIONS AND STELLAR PROPERTIES}

Precision Doppler measurements for the PPPS are obtained with the UCLES echelle spectrograph (Diego et al. 1990) 
Table 1

AAT Radial Velocities for HD 121056

\begin{tabular}{lrc}
\hline \hline JD-2400000 & $\begin{array}{r}\text { Velocity } \\
\left(\mathrm{m} \mathrm{s}^{-1}\right)\end{array}$ & $\begin{array}{r}\text { Uncertainty } \\
\left(\mathrm{m} \mathrm{s}^{-1}\right)\end{array}$ \\
\hline 54866.26766 & -86.6 & 3.3 \\
55318.00434 & -87.0 & 3.2 \\
55318.00981 & -86.2 & 3.4 \\
55380.96409 & -19.8 & 3.2 \\
55382.00821 & -20.7 & 2.8 \\
55580.24855 & -38.0 & 3.3 \\
55602.20623 & 19.8 & 9.8 \\
55602.21786 & 16.3 & 7.1 \\
55602.22211 & 12.5 & 4.6 \\
55907.24206 & 63.0 & 3.3 \\
55969.24861 & 87.6 & 3.0 \\
55969.26001 & 90.7 & 2.9 \\
55971.15907 & 88.1 & 3.1 \\
55994.12084 & 75.1 & 4.8 \\
56051.94440 & 32.8 & 4.0 \\
56088.95971 & 65.0 & 3.3 \\
56344.18468 & 45.0 & 3.5 \\
56376.17506 & -42.2 & 3.2 \\
56378.04416 & -53.2 & 3.1 \\
56400.03556 & -29.2 & 3.4 \\
56527.88115 & -11.1 & 6.3 \\
56745.10350 & -122.1 & 3.4 \\
\hline
\end{tabular}

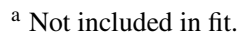

at the $3.9 \mathrm{~m}$ Anglo-Australian Telescope (AAT). The observing procedure is identical to that used by the long-running AngloAustralian Planet Search (e.g., Tinney et al. 2001; Butler et al. 2001; Jones et al. 2010; Wittenmyer et al. 2012); a 1 arcsec slit delivers a resolving power of $R \sim 45,000$. Calibration of the spectrograph point-spread function is achieved using an iodine absorption cell temperature-controlled at $60^{\circ} 0 \pm 0.1 \mathrm{C}$. The iodine cell superimposes a forest of narrow absorption lines from 5000 to $6200 \AA$, allowing simultaneous calibration of instrumental drifts as well as a precise wavelength reference (Valenti et al. 1995; Butler et al. 1996). Velocities are obtained using the Austral code (Endl et al. 2000), which has been successfully used by several planet-search programs for more than $10 \mathrm{yr}$ (Endl et al. 2004; Robertson et al. 2012; Sato et al. 2013).

We have obtained 22 observations of HD 121056 since 2009 February 3, and an iodine-free template spectrum was obtained on 2010 July 4 . With $V=6.19$, exposure times are typically 300-600 s, with a resulting signal-to-noise ratio $(\mathrm{S} / \mathrm{N})$ of 150-300 per pixel each epoch. The data, given in Table 1, span a total of 1879 days $(5.5 \mathrm{yr})$, and have a mean internal velocity uncertainty of $4.0 \mathrm{~m} \mathrm{~s}^{-1}$.

We have used our iodine-free template spectrum $(R \sim 60,000$, $\mathrm{S} / \mathrm{N} \sim 250)$ to derive spectroscopic stellar parameters. In brief, the iron abundance $[\mathrm{Fe} / \mathrm{H}]$ was determined from the equivalent widths (EWs) of 32 unblended Fe lines, and the LTE model atmospheres adopted in this work were interpolated from the ODFNEW grid of ATLAS9 (Castelli \& Kurucz 2004). The effective temperature $\left(T_{\text {eff }}\right)$ and bolometric correction were derived from the color index $B-V$ and the estimated metallicity using the empirical calibration of Alonso et al. (1999, 2001). Since the color- $T_{\text {eff }}$ method is not extinction-free, we corrected for reddening and extinction using $E(B-V)=0.014$ and $A_{V}=3.1 \times E(B-V)=0.043$ (Schlegel et al. 1998). The stellar mass and age were estimated from the interpolation of Yonsei-Yale ( $\mathrm{Y}^{2}$ ) stellar evolution tracks (Yi et al. 2003). The resulting stellar mass of $1.30 \pm 0.18 M_{\odot}$ was adopted for calculating the planet masses. Results from this method are labeled "Method 1" in Table 2.

We also derived stellar parameters with the MOOG program (Sneden 1973), based on the homogeneous, plane-parallel and local thermodynamic equilibrium models (1D-LTE) from Castelli \& Kurucz (2003). The program matches the observed EWs with theoretical values calculated based on the atmospheric model. We obtained the effective temperature by forcing a consistent iron abundance, derived from $81 \mathrm{Fe}$ I lines with their excitation potentials. We determine $\log g$ by forcing the Fe I and $\mathrm{Fe}$ II lines to give the same iron abundance. The microturbulent velocity $v_{t}$ is determined by requiring a zero-slope relation between the log of the iron abundance and the EWs. The results from this method are labeled "Method 2" in Table 2.

\section{ORBIT FITTING AND PLANETARY PARAMETERS}

We fit for the planets in two ways: first, using only the AAT observations as a wholly independent check of Jones et al. (2014), and then we include the published velocities for a joint solution.

\subsection{AAT/UCLES Data Only}

The 22 AAT velocities for HD 121056 have an rms scatter of $64 \mathrm{~m} \mathrm{~s}^{-1}$ and showed an obvious trend after $\sim 2$ yr. The trend

Table 2

Stellar Parameters for HD 121056

\begin{tabular}{|c|c|c|c|c|}
\hline Parameter & Method 1 & Method 2 & Literature & Reference \\
\hline Spec. type & & & K0 III & Houk (1982) \\
\hline Distance (pc) & & & $66.0 \pm 1.7$ & van Leeuwen (2007) \\
\hline$(B-V)$ & & & $1.008 \pm 0.014$ & van Leeuwen (2007) \\
\hline Mass $\left(M_{\odot}\right)$ & $1.30 \pm 0.18$ & 1.21 & $\begin{array}{c}1.63 \pm 0.22 \\
1.4\end{array}$ & $\begin{array}{l}\text { Jones et al. (2014) } \\
\text { Randich et al. (1999) }\end{array}$ \\
\hline $\mathrm{V} \sin i\left(\mathrm{~km} \mathrm{~s}^{-1}\right)$ & $<2$ & & $\begin{array}{c}1.4 \\
1.8 \pm 0.9\end{array}$ & $\begin{array}{l}\text { Randich et al. (1999) } \\
\text { Jones et al. (2011) }\end{array}$ \\
\hline$[\mathrm{Fe} / H]$ & $-0.03 \pm 0.10$ & $-0.13 \pm 0.06$ & $\begin{aligned}-0.11 & \pm 0.09 \\
0.00 & \pm 0.10\end{aligned}$ & $\begin{array}{l}\text { Randich et al. (1999) } \\
\text { Jones et al. (2011) }\end{array}$ \\
\hline$T_{\text {eff }}(\mathrm{K})$ & $4805 \pm 100$ & $4859 \pm 100$ & $\begin{array}{l}4711 \pm 100 \\
4890 \pm 100\end{array}$ & $\begin{array}{l}\text { Randich et al. (1999) } \\
\text { Jones et al. (2014) }\end{array}$ \\
\hline $\log g$ & $3.04 \pm 0.10$ & $2.89 \pm 0.10$ & $\begin{array}{c}3.0 \pm 0.3 \\
3.15 \pm 0.20\end{array}$ & $\begin{array}{l}\text { Randich et al. (1999) } \\
\text { Jones et al. (2014) }\end{array}$ \\
\hline$v_{t}\left(\mathrm{~km} \mathrm{~s}^{-1}\right)$ & $1.35 \pm 0.09$ & $1.21 \pm 0.08$ & & \\
\hline Radius $\left(R_{\odot}\right)$ & $5.87 \pm 0.29$ & & $5.92 \pm 0.44$ & Jones et al. (2014) \\
\hline Luminosity $\left(L_{\odot}\right)$ & $16.1 \pm 0.8$ & 18.2 & $17.6 \pm 2.6$ & Jones et al. (2014) \\
\hline
\end{tabular}



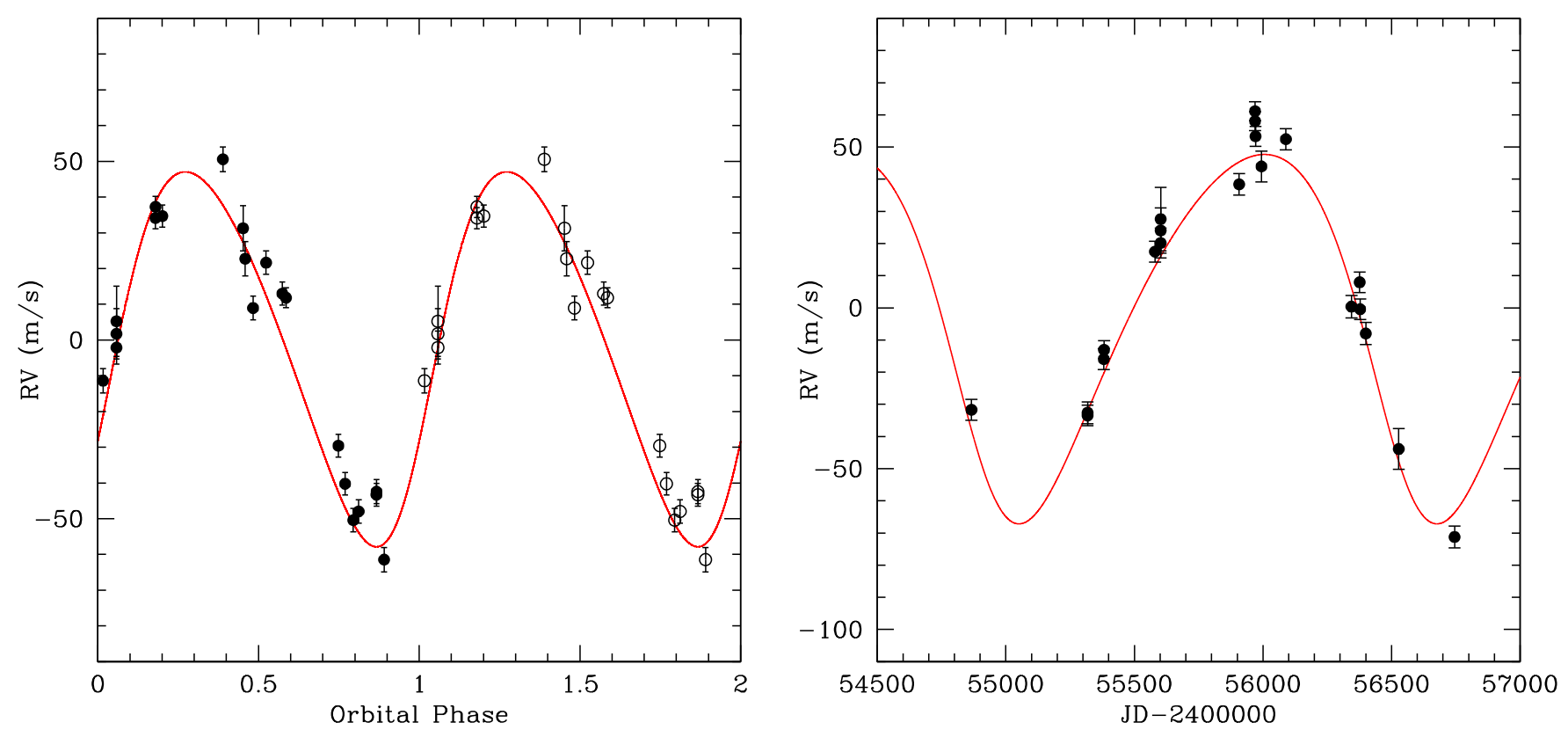

Figure 1. AAT data and model fit for the HD 121056 planets. Left panel: phase plot and model fit for HD 121056b, with the outer planet removed. Two cycles are shown for clarity. Right panel: radial-velocity time series for HD $121056 \mathrm{c}$, with the inner planet removed. The rms about the two-planet fit is $3.2 \mathrm{~m} \mathrm{~s}^{-1}$.

Table 3

HD 121056 Planetary System Parameters (AAT Data Only)

\begin{tabular}{lccccc}
\hline \hline Parameter & \multicolumn{2}{c}{ GaussFit Solution } & & \multicolumn{2}{c}{ Bootstrap Solution $^{\mathrm{a}}$} \\
\cline { 2 - 3 } & HD 121056b & HD 121056c & & HD 121056b & HD 121056c \\
\hline Period (days) & $89.06 \pm 0.10$ & $1626 \pm 26$ & & $88.99 \pm 0.33$ & $1653 \pm 132$ \\
Eccentricity & $0.17 \pm 0.04$ & $0.20 \pm 0.05$ & & $0.34 \pm 0.19$ & $0.34 \pm 0.18$ \\
$\omega(\mathrm{deg})$ & $244 \pm 10$ & $136 \pm 11$ & & $213 \pm 38$ & $155 \pm 54$ \\
$K\left(\mathrm{~m} \mathrm{~s}^{-1}\right)$ & $52.5 \pm 2.2$ & $57.4 \pm 3.1$ & & $60.2 \pm 11.0$ & $70.5 \pm 17.5$ \\
$T_{0}(\mathrm{JD}-2400000)$ & $55329.8 \pm 2.6$ & $53290 \pm 59$ & & $54785 \pm 12$ & $53308 \pm 248$ \\
$\mathrm{~m} \sin i\left(\mathrm{M}_{\mathrm{Jup}}\right)$ & $1.35 \pm 0.17$ & $3.88 \pm 0.55$ & & $1.48 \pm 0.30$ & $4.60 \pm 1.37$ \\
$a($ AU) & $0.426 \pm 0.020$ & $2.96 \pm 0.16$ & & $0.426 \pm 0.021$ & $2.99 \pm 0.27$ \\
\hline rms of fit $\left(\mathrm{m} \mathrm{s}^{-1}\right)$ & 3.23 & & 3.16 & \\
$\chi_{v}^{2}$ & 1.36 & & 1.36 & \\
\hline
\end{tabular}

Note. ${ }^{a}$ Mean parameter value and $68.7 \%$ confidence interval from 10,000 bootstrap iterations.

turned over in early 2013 , and could be tentatively fit with a long-period object, though the rms scatter remained stubbornly high $\left(\sim 30 \mathrm{~m} \mathrm{~s}^{-1}\right)$ compared to the mean velocity uncertainty of $4.0 \mathrm{~m} \mathrm{~s}^{-1}$ for this bright star. Due to the limited amount of data available, we used a genetic algorithm (Charbonneau 1995) rather than a traditional Lomb-Scargle periodogram search (Lomb 1976; Scargle 1982). We have successfully used this technique in previous work (e.g., Tinney et al. 2011; Wittenmyer et al. 2011a; Horner et al. 2012) to detect planetary signals when data are sparse or when the candidate orbital periods are highly uncertain. To check the results of Jones et al. (2014), we allowed both planets to take on a very wide range of orbital periods ( $P_{1}:$ :40-500 days, $P_{2}: 1200-2500$ days), and we ran the genetic algorithm for 50,000 iterations, testing a total of about $10^{7}$ possible configurations. Convergence rapidly occurred, on a solution in which the inner planet is consistent with the result of Jones et al. (2014), and the outer planet has a period $P \sim 1650$ days with low eccentricity.

The best two-planet solution was then used as a starting point for the generalized least-squares program GaussFit (Jefferys et al. 1988), here used to solve a Keplerian radial-velocity orbit model. For the final fitting, we excluded the velocity point from JD 2456051, as it lay $5 \sigma$ from the model and was obtained in exceedingly poor seeing $\left(\sim 4^{\prime \prime}\right)$. The rms about the two-planet model was $6.4 \mathrm{~m} \mathrm{~s}^{-1}$, but dropped to $3.2 \mathrm{~m} \mathrm{~s}^{-1}$ after removing that point. The data and model fit are shown in Figure 1, and the best-fit system parameters are given in Table 3. Since the formal uncertainties derived from the covariance matrix may be underestimated, we also estimated parameter uncertainties using a bootstrap randomization method within the Systemic Console (Meschiari et al. 2009), which generates 10,000 simulated data sets by drawing with replacement from the original data. Since the chief constraint on the outer planet comes from the first data point (at JD 2454866), the bootstrap method provides more realistic (and much larger) uncertainty estimates, particularly for the orbital period and mass. The rightmost two columns of Table 3 give the mean value and $1 \sigma$ uncertainties for each parameter from the bootstrapping.

\subsection{Combined Fit with Published Data}

We repeated the above fitting procedures, now including the 60 velocities from FEROS and CHIRON as published in Jones et al. (2014). The results are given in Table 4. The rms scatter about the fit for the three data sets is as follows: AAT- $9.26 \mathrm{~m} \mathrm{~s}^{-1}$, FEROS-11.02 $\mathrm{m} \mathrm{s}^{-1}$, CHIRON$9.85 \mathrm{~m} \mathrm{~s}^{-1}$. This scatter is considerably higher, but consistent 
Table 4

HD 121056 Planetary System Parameters (All Data)

\begin{tabular}{|c|c|c|c|c|}
\hline \multirow[t]{2}{*}{ Parameter } & \multicolumn{2}{|c|}{ GaussFit Solution } & \multicolumn{2}{|c|}{ Bootstrap Solution $^{\mathrm{a}}$} \\
\hline & HD $121056 b$ & HD $121056 \mathrm{c}$ & HD $121056 b$ & HD $121056 \mathrm{c}$ \\
\hline Period (days) & $89.09 \pm 0.12$ & $1741 \pm 39$ & $89.09 \pm 0.11$ & $2203 \pm 486$ \\
\hline Eccentricity & $0.02 \pm 0.04$ & $0.20 \pm 0.04$ & $0.06 \pm 0.04$ & $0.18 \pm 0.07$ \\
\hline$\omega(\operatorname{deg})$ & $211 \pm 103$ & $201 \pm 9$ & $300 \pm 132$ & $205 \pm 17$ \\
\hline$K\left(\mathrm{~m} \mathrm{~s}^{-1}\right)$ & $47.9 \pm 1.8$ & $62.8 \pm 2.8$ & $47.9 \pm 11.0$ & $81.9 \pm 14.0$ \\
\hline$T_{0}(\mathrm{JD}-2400000)$ & $55500 \pm 26$ & $53427 \pm 56$ & $54810 \pm 33$ & $53068 \pm 405$ \\
\hline $\mathrm{m} \sin i\left(\mathrm{M}_{\mathrm{Jup}}\right)$ & $1.25 \pm 0.16$ & $4.34 \pm 0.59$ & $1.25 \pm 0.04$ & $6.14 \pm 1.99$ \\
\hline$a(\mathrm{AU})$ & $0.426 \pm 0.020$ & $3.09 \pm 0.18$ & $0.426 \pm 0.020$ & $3.62 \pm 0.58$ \\
\hline rms of fit-AAT $\left(\mathrm{m} \mathrm{s}^{-1}\right)$ & 9.26 & & 9.27 & \\
\hline rms of fit-FEROS $\left(\mathrm{m} \mathrm{s}^{-1}\right)$ & 11.02 & & 11.01 & \\
\hline rms of fit-CHIRON $\left(\mathrm{m} \mathrm{s}^{-1}\right)$ & 9.85 & & 9.85 & \\
\hline$\chi_{v}^{2}$ & 5.46 & & 5.46 & \\
\hline
\end{tabular}

Note. ${ }^{a}$ Mean parameter value and $68.7 \%$ confidence interval from 10,000 bootstrap iterations.

with the expected pulsation-induced velocity jitter, as demonstrated by Endl et al. (2009) for Gamma Cephei, a giant star of similar evolutionary status as HD 121056. The combined fit gives a near-zero eccentricity for the inner planet and a somewhat longer period for the outer planet. The bootstrap process favors a period near 2200 days (with a large uncertainty of 486 days), similar to the 2100 days estimate of Jones et al. (2014) and statistically consistent with the shorter periods given in Table 3 . The parameter distributions obtained from the 10,000 bootstrap trials are shown in Figure 2 (inner planet) and Figure 3 (outer planet). Most importantly, all the fitting permutations described in this section unambiguously confirm the presence of the 89 day inner planet and the planetary nature of the outer companion, even when accounting for the high leverage of the first observation.

\section{DISCUSSION}

\subsection{Evidence for Orbiting Planets}

For any planet discovery, it is important to check for the possibility that the observed radial-velocity variations are intrinsic to the star (or the instrument) and not due to orbiting planets. For HD 121056, the periods of the two signals (89 and $\sim 1700$ days) are nowhere near the window function peak at $\sim 21$ days, nor are they near $1 \mathrm{yr}$. Spurious periods in observational data most commonly arise at those periods due to sampling (imposed by the lunar cycle and yearly observability of a given target). We also note that there are no large phase gaps in the data (Figure 1) - such gaps would also cast doubt on the reality of a signal. There are also no potentially contaminating background objects within 5 arcmin of HD 121056.

The chromospheric activity index $S_{\mathrm{HK}}$, determined from observations of the $\mathrm{Ca}$ II $\mathrm{H}$ and $\mathrm{K}$ lines, is a critically important measurement for radial-velocity planet detection, as the star's activity is usually correlated with velocity variations that can mimic the reflex velocities induced by orbiting planets (e.g., Bonfils et al. 2007; Hatzes et al. 2010). Jones et al. (2014) demonstrated that the $S_{\mathrm{HK}}$ index was not correlated to their velocities, supporting the planet hypothesis. For radial-velocity detected planets, a major concern is that the signal is due to rotational modulation of starspots. For some stars, particularly giants, spots can induce quasi-periodic velocity variations in excess of $20 \mathrm{~m} \mathrm{~s}^{-1}$ (Hekker et al. 2008). For a spotted star, the rotation period can be deduced from photometry; Jones et al. (2014) found no significant periodicities in Hipparcos photom- etry, and no correlations between the radial velocities and the line profiles as measured in the bisector velocity span and the cross-correlation FWHM. Following Wittenmyer et al. (2011a), we can combine the available estimates of the star's radius and $\mathrm{v}$ sin $i$ (Table 2) minimum rotational velocity to obtain a maximum rotation period. This calculation yields a maximum rotation period of $167 \pm 83$ days, which is distinctly different from either candidate planet's orbital period. Furthermore, if the 89 day signal were caused by spots, it seems exceedingly unlikely that one or more starspots would persist so coherently over the $5.5 \mathrm{yr}$ of our observations such that we detect the clean and well-sampled signal presented here (Figure 1). These lines of evidence lead us to conclude that the simplest explanation for the observed highly significant and coherent radialvelocity variations is the gravitational influence of two orbiting giant planets.

\subsection{Dynamical Considerations}

There is a growing body of recent work calling for the rigorous dynamical stability testing of proposed multiple-planet systems (e.g., Horner et al. 2011; Hinse et al. 2012; Wittenmyer et al. 2013a; Horner et al. 2013). Detailed dynamical testing can confirm or refute the orbital configurations inferred from Keplerian fits, and hence is a valuable part of the discovery process.

In many cases, when the planets proposed are relatively tightly packed, dynamically speaking, the only way to address the question of their stability is to run large scale suites of dynamical simulations, such as those presented in Wittenmyer et al. (2013c). In some cases, however, the proposed planets are so widely spaced that it is highly unlikely that they would interact with one another sufficiently strongly to disrupt the system. Such planets are essentially decoupled from one another (e.g., HD 159868b,c; Wittenmyer et al. 2012).

Gladman (1993) found that, for planets moving on orbits with low eccentricity and inclination, two planet systems are typically stable when the orbits of the planets are separated by more than $2 \sqrt{3}$ times their mutual Hill radius, where the mutual Hill radius is defined as

$$
R_{H}=\left[\frac{\left(m_{1}+m_{2}\right)}{3 M_{\odot}}\right]^{1 / 3}\left[\frac{\left(a_{1}+a_{2}\right)}{2}\right] .
$$

Chambers et al. (1996) tested this using numerical integrations, and confirmed that this result holds, in general. As such, this 

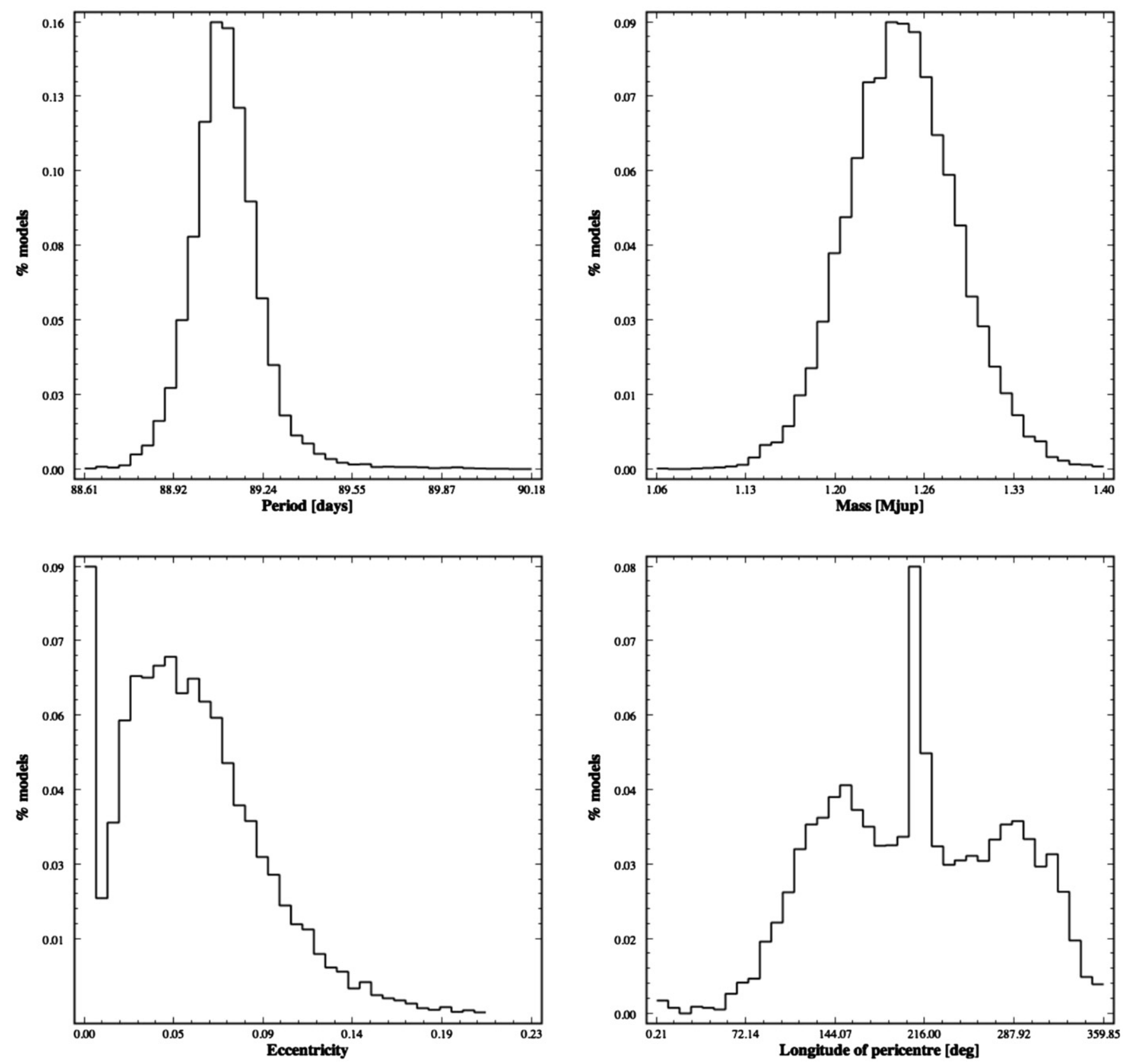

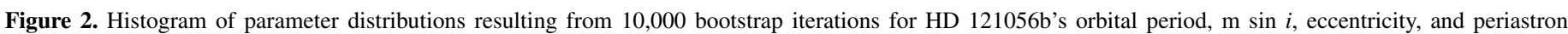
argument $\omega$. All velocity data were used.

seems a reasonable first criterion by which the potential stability of which a given two-planet system can be considered. Clearly, the greater the separation of a given two planet system, measured in Hill radii, the more likely it is to be truly dynamically stable.

In the case of the HD 121056 system, Equation (1) reveals that the mutual Hill radius of the two planets proposed in this work is $R_{H} \sim 0.265 \mathrm{AU}$. The orbits of the planets are thus separated by $\sim 9.6$ mutual Hill radii-far more widely spaced than the $2 \sqrt{3} R_{H}$ stability criterion of Gladman (1993). Indeed, the planets are sufficiently widely spaced that the system is likely to have dynamical room for at least one additional planet between their orbits. Our AAT data, with a residual rms scatter of only $3.2 \mathrm{~m} \mathrm{~s}^{-1}$, can be used to place reasonably tight limits on the presence of any undetected planet. In brief, we add the
Keplerian velocity signal of a fictitious planet to the residuals of our fit, and attempt to recover it via a generalized Lomb-Scargle periodogram (Zechmeister \& Kürster 2009). Here, we have assumed circular orbits; for each combination of period $P$ and radial-velocity semiamplitude $K$, we tried 30 values of orbital phase. A planet is deemed detectable if $99 \%$ of orbital configurations at a given $P$ and $K$ are recovered with a falsealarm probability (Sturrock \& Scargle 2010) of less than $1 \%$. This approach is essentially identical to that used in our previous work (e.g., Wittenmyer et al. 2006, 2011b, 2013b). Using the AAT-only fit residuals, we can with $99 \%$ confidence exclude planets with $\mathrm{m} \sin i>0.41 \mathrm{M}_{\mathrm{Jup}}$ on circular orbits between the orbital excursions of the two planets $(0.5<a<2.3 \mathrm{AU})$. Including all available data as in Section 3.2, we obtain a mass limit of $\mathrm{m} \sin i>0.66 \mathrm{M}_{\mathrm{Jup}}$. Further observations over additional 

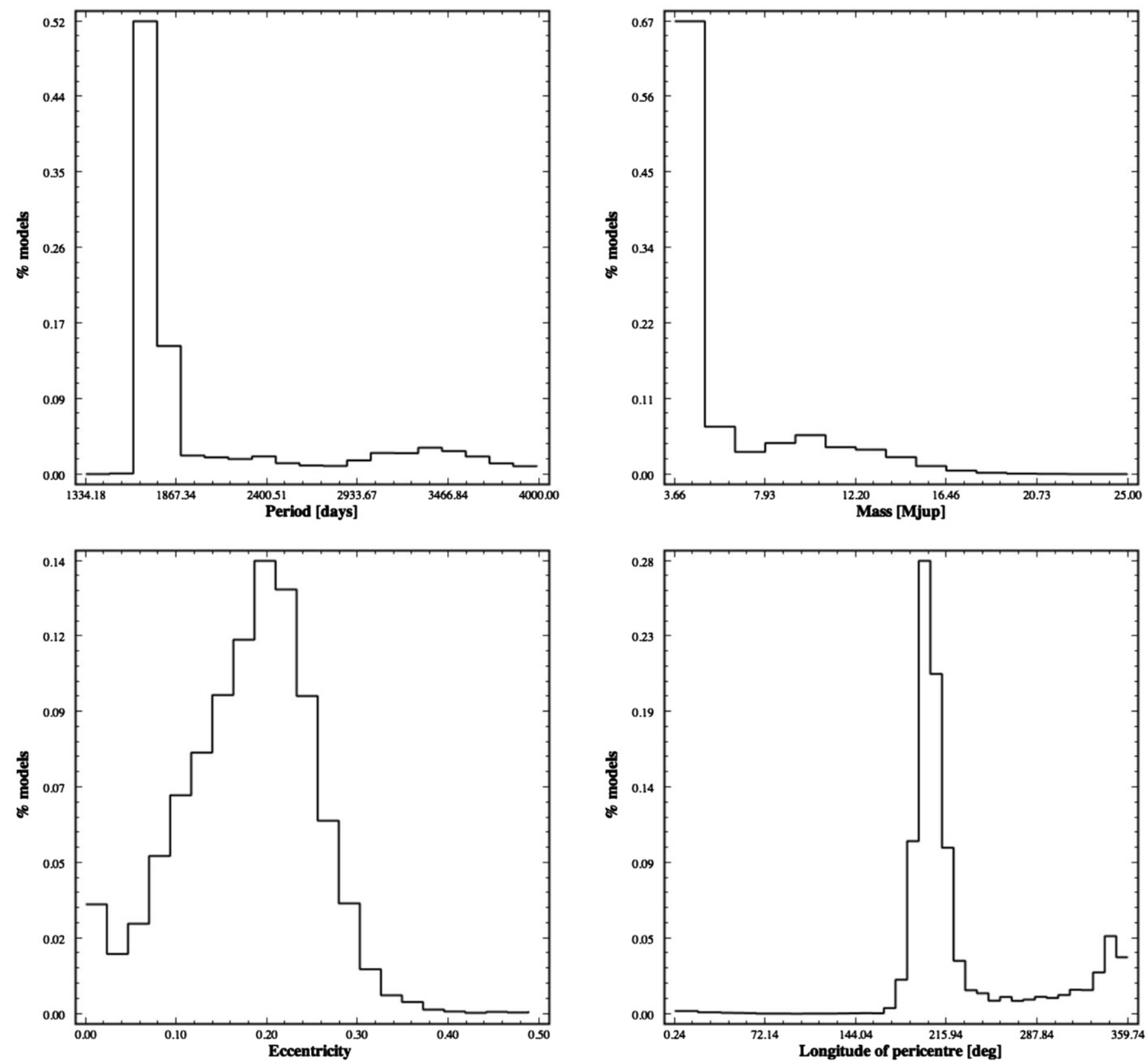

Figure 3. Same as Figure 2, but for HD 121056c. The best-fit period for the outer planet relies critically on the first epoch, as demonstrated by the long tail in the period and mass distributions.

orbital cycles of the outer planet will substantially improve the quality of the two-Keplerian fit and better constrain the region between the planets.

\subsection{Conclusions}

We have independently confirmed the existence of a system of two giant planets orbiting the K0 giant HD 121056 (Jones et al. 2014). Such confirmation is essential as the system hosts a close-in planet $(a<0.5 \mathrm{AU})$, a rarity among giant stars. The longer baseline of our data also confirm the planetary nature of the outer companion proposed by Jones et al. (2014). The multiplicity of the HD 121056 system makes it stand out from the growing crowd of planets known to orbit evolved stars. Only six multiple-planet systems have been found around giant stars: HD 4732 (Sato et al. 2013), HD 200964 and 24 Sex
(Johnson et al. 2011b), Kepler-391 (Rowe et al. 2014), Kepler-56 (Huber et al. 2013), and BD+20 2457 (Niedzielski et al. 2009b). However, the latter system has been shown to be dynamically unstable (Horner et al. 2014), casting doubt on its veracity. If we consider the surface gravity $\log g$ as a proxy for the degree to which a star has evolved off the main sequence, HD 121056 is the most-evolved star to host a multiple-planet system (Figure 4). With this in mind, the lower eccentricity for HD $121056 \mathrm{~b}$ found by the combined fit (Table 4) seems more physically plausible, arising from tidal circularization interactions with the expanding host star. The fate of planets as their host stars evolve have been modeled in detail (e.g., Villaver \& Livio 2009; Kunitomo et al. 2011; Mustill et al. 2014). Unsurprisingly, at $a=0.426 \mathrm{AU}, \mathrm{HD} 121056 \mathrm{~b}$ is doomed to be engulfed. The fate of HD 121056c is less clear: models by Villaver \& Livio (2007) show that for a $1 M_{\odot}$ star, planets beyond $a \sim 3 \mathrm{AU}$ are 


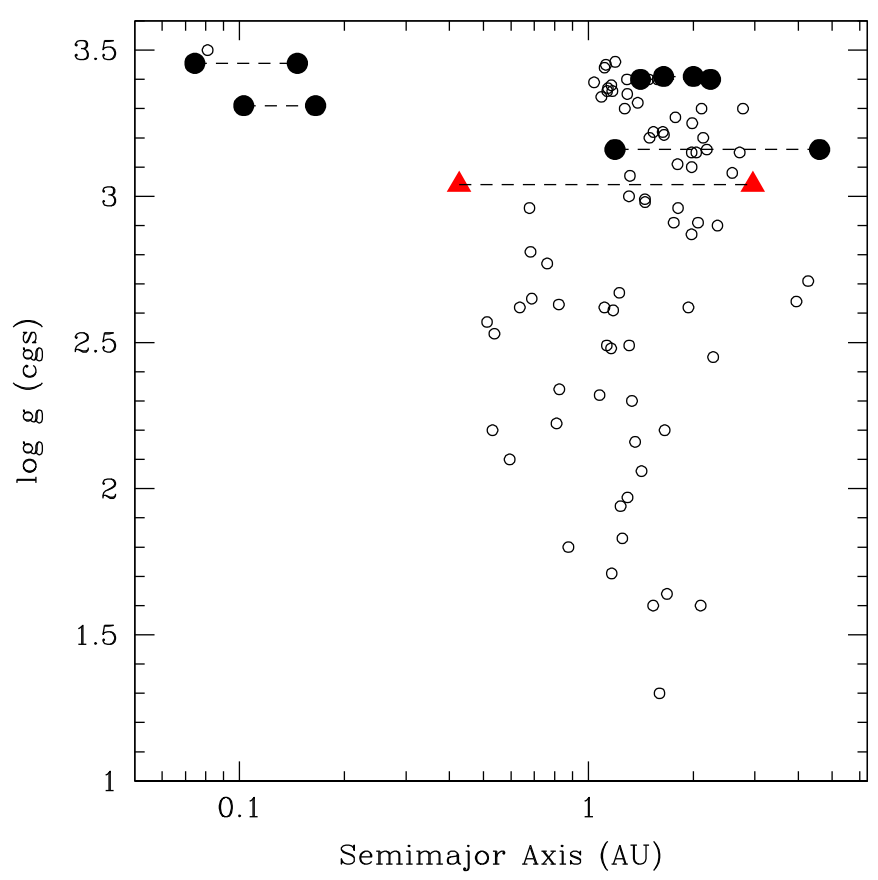

Figure 4. Confirmed planets orbiting giant stars $(\log g<3.5)$. Planets in multiple systems are shown as filled circles connected by a dashed line. BD+20 2457 (Niedzielski et al. 2009b) is not shown as it was demonstrated to be dynamically unfeasible by Horner et al. (2014). HD 121056b and c are shown as red triangles.

likely to survive the asymptotic giant branch phase. However, Villaver \& Livio (2009) showed that a $5 \mathrm{M}_{\text {Jup }}$ planet must have $a \gtrsim 3.7$ AU to avoid tidal capture in the red-giant phase. Mustill \& Villaver (2012) likewise find the minimum orbital distance for survival to be $\sim 2.6 \mathrm{AU}$, increasing for eccentric planets. The significant uncertainties in the eccentricity and semimajor axis of HD 121056c mean that its fate rests on a knife edge, to be crystallized by future observations refining its orbit.

The PPPS aimed to explore the dependence of planetary system properties on host-star mass by improve the detection statistics for intermediate-mass stars $\left(1.5-3.0 M_{\odot}\right)$. Many of the other programs listed in Section 1 are likewise targeting evolved stars to explore the same parameter space-though the masses of such stars have been put into question (e.g., Lloyd 2011, 2013; Johnson et al. 2013, 2014). While HD 121056 is most likely a near-solar-mass star (Table 2), its two super-Jovian mass planets are characteristic of those planets orbiting higher-mass stars. That is, planets orbiting stars more massive than the Sun tend to be more massive (Bowler et al. 2010) and move on more circular orbits (Johnson 2008).

By good fortune, our data for HD 121056 sampled the inner planet's phase well enough to enable secure detection with relatively few observations. Jones et al. (2014) were able to obtain high-cadence CHIRON observations to confirm the inner planet. This example highlights the need for high-cadence monitoring of evolved stars to determine whether the deficit of close-in planets (e.g., Figure 4) is real or an observational bias arising from highly competitive and sparsely scheduled time on large telescopes. Dedicated exoplanet observatories such as the Automated Planet Finder (Vogt et al. 2014) or MINERVA (Wright et al. 2014; Swift et al. 2014) are the way forward. Further opportunities can be found with smaller telescopes equipped with high-resolution spectrographs for precise Doppler velocimetry, e.g., New Zealand's Mount John University Observatory (Hearnshaw et al. 2002; Endl et al. 2014) and Shandong University's Weihai Observatory (Gao \& Ren 2014; Cao et al. 2014).

C.G.T. is supported by Australian Research Council grants DP0774000 and DP130102695. J.H. and B.D.C. are supported by USQ's Strategic Research Fund. We gratefully acknowledge the efforts of PPPS guest observers Hugh Jones and Simon O'Toole. This research has made use of NASA's Astrophysics Data System (ADS), and the SIMBAD database, operated at CDS, Strasbourg, France. This research has also made use of the Exoplanet Orbit Database and the Exoplanet Data Explorer at exoplanets.org (Wright et al. 2011). M.E. is supported by the National Science Foundation through grant AST-1313075.

\section{REFERENCES}

Alonso, A., Arribas, S., \& Martínez-Roger, C. 1999, A\&AS, 140, 261 Alonso, A., Arribas, S., \& Martínez-Roger, C. 2001, A\&A, 376, 1039 Barnes, J. R., Jenkins, J. S., Jones, H. R. A., et al. 2012, MNRAS, 424, 591 Batalha, N. M., Rowe, J. F., Bryson, S. T., et al. 2013, ApJS, 204, 24 Bean, J. L., Seifahrt, A., Hartman, H., et al. 2010, ApJ, 713, 410 Bonfils, X., Delfosse, X., Udry, S., et al. 2013, A\&A, 549, A109 Bonfils, X., Mayor, M., Delfosse, X., et al. 2007, A\&A, 474, 293 Borucki, W. J., Koch, D., Basri, G., et al. 2010, Sci, 327, 977

Bowler, B. P., Johnson, J. A., Marcy, G. W., et al. 2010, ApJ, 709, 396 Butler, R. P., Marcy, G. W., Williams, E., et al. 1996, PASP, 108, 500 Butler, R. P., Tinney, C. G., Marcy, G. W., et al. 2001, ApJ, 555, 410

Cao, C., Ren, D., Gao, D., et al. 2014, in IAU Symp. 293, Formation, Detection, and Characterization of Extrasolar Habitable Planets, ed. N. Haghighipour \& J.-L. Zhou (Cambridge: Cambridge Univ. Press), 33

Castelli, F., \& Kurucz, R. L. 2003, in proc. IAU Symp. 210, Modelling of Stellar Atmospheres, ed. Piskunov et al. (Dordrecht: Kluwer), A20

Castelli, F., \& Kurucz, R. L. 2004, in Proc. IAU Symp. 210, Modelling of Stellar Atmospheres, ed. N. Piskunov et al. (Dordrecht: Kluwer), A20 (arXiv:astroph/0405087)

Chambers, J. E., Wetherill, G. W., \& Boss, A. P. 1996, Icar, 119, 261

Charbonneau, P. 1995, ApJS, 101, 309

Ciceri, S., Lillo-Box, J., Southworth, J., et al. 2014, A\&A, 573, L5

Diego, F., Charalambous, A., Fish, A. C., \& Walker, D. D. 1990, Proc. Soc. Photo-Opt. Instr. Eng., 1235, 562

Döllinger, M. P., Hatzes, A. P., Pasquini, L., et al. 2007, A\&A, 472, 649

Endl, M., Bean, J. L., Wittenmyer, R. A., et al. 2009, in AIP Conf. Proc. 1170, Stellar Pulsation: challenges for Theory and Observation, ed. J. A. Guzik \& P. A. Bradley (Melville, NY: AIP), 543

Endl, M., Bergmann, C., Hearnshaw, J., et al. 2014, IJAsB, in press (arXiv:1403.4809)

Endl, M., Cochran, W. D., Kürster, M., et al. 2006, ApJ, 649, 436

Endl, M., Hatzes, A. P., Cochran, W. D., et al. 2004, ApJ, 611, 1121

Endl, M., Kürster, M., \& Els, S. 2000, A\&A, 362, 585

Galland, F., Lagrange, A.-M., Udry, S., et al. 2005, A\&A, 443, 337

Gao, D., \& Ren, D. 2014, in IAU Symp. 293, Formation, Detection, and Characterization of Extrasolar Habitable Planets, ed. N. Haghighipour \& J.-L. Zhou (Cambridge: Cambridge Univ. Press), 400

Gladman, B. 1993, Icar, 106, 247

Hatzes, A. P., Dvorak, R., Wuchterl, G., et al. 2010, A\&A, 520, A93

Hatzes, A. P., Guenther, E. W., Endl, M., et al. 2005, A\&A, 437, 743

Hearnshaw, J. B., Barnes, S. I., Kershaw, G. M., et al. 2002, ExA, 13, 59

Hekker, S., Snellen, I. A. G., Aerts, C., et al. 2008, JPhCS, 118, 012058

Hinse, T. C., Goździewski, K., Lee, J. W., Haghighipour, N., \& Lee, C.-U. 2012, AJ, 144, 34

Horner, J., Marshall, J. P., Wittenmyer, R. A., \& Tinney, C. G. 2011, MNRAS, 416, L11

Horner, J., Wittenmyer, R. A., Hinse, T. C., \& Marshall, J. P. 2014, MNRAS, 439, 1176

Horner, J., Wittenmyer, R. A., Hinse, T. C., \& Tinney, C. G. 2012, MNRAS, 425,749

Horner, J., Wittenmyer, R. A., Hinse, T. C., et al. 2013, MNRAS, 435, 2033

Houk, N. 1982, in Michigan Catalogue of Two-dimensional Spectral Types for the HD stars. Volume_3. Declinations -40 to -26 ., ed. N. Houk (Ann Arbor, MI: Department of Astronomy, Univ. Michigan), 390

Huber, D., Carter, J. A., Barbieri, M., et al. 2013, Sci, 342, 331

Jefferys, W. H., Fitzpatrick, M. J., \& McArthur, B. E. 1988, CeMec, 41, 39 
Johnson, J. A. 2008, in ASP Conf. Ser. 398, Extreme Solar Systems, ed. D. Fischer, F. A. Rasio, S. E. Thorsett, \& A. Wolszczan (San Francisco, CA: ASP), 59

Johnson, J. A., Bowler, B. P., Howard, A. W., et al. 2010a, ApJL, 721, L153

Johnson, J. A., Clanton, C., Howard, A. W., et al. 2011a, ApJS, 197, 26

Johnson, J. A., Fischer, D. A., Marcy, G. W., et al. 2007, ApJ, 665, 785

Johnson, J. A., Howard, A. W., Marcy, G. W., et al. 2010b, PASP, 122, 149

Johnson, J. A., Huber, D., Boyajian, T., et al. 2014, ApJ, 794, 15

Johnson, J. A., Marcy, G. W., Fischer, D. A., et al. 2006, ApJ, 652, 1724

Johnson, J. A., Morton, T. D., \& Wright, J. T. 2013, ApJ, 763, 53

Johnson, J. A., Payne, M., Howard, A. W., et al. 2011b, AJ, 141, 16

Jones, H. R. A., Butler, R. P., Tinney, C. G., et al. 2010, MNRAS, 403, 1703

Jones, M. I., Jenkins, J. S., Rojo, P., \& Melo, C. H. F. 2011, A\&A, 536, AA71 Jones, M. I., Jenkins, J. S., Rojo, P., Melo, C. H. F., \& Bluhm, P. 2014, A\&A, 573, A3

Kunitomo, M., Ikoma, M., Sato, B., Katsuta, Y., \& Ida, S. 2011, ApJ, 737, 66

Lillo-Box, J., Barrado, D., Moya, A., et al. 2014, A\&A, 562, AA109

Lloyd, J. P. 2011, ApJL, 739, LL49

Lloyd, J. P. 2013, ApJL, 774, LL2

Lomb, N. R. 1976, Ap\&SS, 39, 447

Mahadevan, S., Ramsey, L., Bender, C., et al. 2012, Proc. SPIE, 8446, 84461S

Meschiari, S., Wolf, A. S., Rivera, E., et al. 2009, PASP, 121, 1016

Mortier, A., Santos, N. C., Sousa, S. G., et al. 2013, A\&A, 557, A70

Mustill, A. J., Veras, D., \& Villaver, E. 2014, MNRAS, 437, 1404

Mustill, A. J., \& Villaver, E. 2012, ApJ, 761, 121

Niedzielski, A., Goździewski, K., Wolszczan, A., et al. 2009a, ApJ, 693, 276

Niedzielski, A., Nowak, G., Adamów, M., \& Wolszczan, A. 2009b, ApJ, 707, 768

Quirrenbach, A., Amado, P. J., Mandel, H., et al. 2010, Proc. SPIE, 7735, 773513

Randich, S., Gratton, R., Pallavicini, R., Pasquini, L., \& Carretta, E. 1999, A\&A, 348, 487

Robertson, P., Endl, M., Cochran, W. D., et al. 2012, ApJ, 749, 39

Rowe, J. F., Bryson, S. T., Marcy, G. W., et al. 2014, ApJ, 784, 45

Sato, B., Kambe, E., Takeda, Y., et al. 2005, PASJ, 57, 97
Sato, B., Omiya, M., Liu, Y., et al. 2010, PASJ, 62, 1063

Sato, B., Omiya, M., Wittenmyer, R. A., et al. 2013, ApJ, 762, 9

Scargle, J. D. 1982, ApJ, 263, 835

Schlegel, D. J., Finkbeiner, D. P., \& Davis, M. 1998, ApJ, 500, 525

Setiawan, J., Pasquini, L., da Silva, L., von der Lühe, O., \& Hatzes, A. 2003, A\&A, 397, 1151

Sneden, C. A. 1973, PhD thesis, Univ. of Texas at Austin

Sturrock, P. A., \& Scargle, J. D. 2010, ApJ, 718, 527

Swift, J. J., Bottom, M., Johnson, J. A., et al. 2014, JATIS, submitted (arXiv:1411.3724)

Tinney, C. G., Butler, R. P., Marcy, G. W., et al. 2001, ApJ, 551, 507

Tinney, C. G., Wittenmyer, R. A., Butler, R. P., et al. 2011, ApJ, 732, 31

Valenti, J. A., Butler, R. P., \& Marcy, G. W. 1995, PASP, 107, 966

van Leeuwen, F. 2007, A\&A, 474, 653

Villaver, E., \& Livio, M. 2007, ApJ, 661, 1192

Villaver, E., \& Livio, M. 2009, ApJL, 705, L81

Villaver, E., Livio, M., Mustill, A. J., \& Siess, L. 2014, ApJ, 794, 3

Vogt, S. S., Radovan, M., Kibrick, R., et al. 2014, PASP, 126, 359

Wittenmyer, R. A., Endl, M., Cochran, W. D., et al. 2006, AJ, 132, 177

Wittenmyer, R. A., Endl, M., Wang, L., et al. 2011a, ApJ, 743, 184

Wittenmyer, R. A., Horner, J., \& Marshall, J. P. 2013a, MNRAS, 431, 2150

Wittenmyer, R. A., Horner, J., Tuomi, M., et al. 2012, ApJ, 753, 169

Wittenmyer, R. A., Tinney, C. G., Horner, J., et al. 2013b, PASP, 125, 351

Wittenmyer, R. A., Tinney, C. G., O’Toole, S. J., et al. 2011b, ApJ, 727, 102

Wittenmyer, R. A., Wang, S., Horner, J., et al. 2013c, ApJS, 208, 2

Wright, D. J., Tinney, C. G., \& Wittenmyer, R. A. 2014, in IAU Symp. 293, Formation, Detection, and Characterization of Extrasolar Habitable Planets, ed. N. Haghighipour \& J.-L. Zhou (Cambridge: Cambridge Univ. Press), 68

Wright, J., Johnson, J. A., McCrady, N., et al. 2014, American Astronomical Society Meeting Abstracts, 223, 148

Wright, J. T., Fakhouri, O., Marcy, G. W., et al. 2011, PASP, 123, 412

Yi, S. K., Kim, Y.-C., \& Demarque, P. 2003, ApJS, 144, 259

Zechmeister, M., \& Kürster, M. 2009, A\&A, 496, 577 